\title{
Predictions of local ground geomagnetic field fluctuations during the 7-10 November 2004 events studied with solar wind driven models
}

\author{
P. Wintoft ${ }^{1}$, M. Wik ${ }^{1}$, H. Lundstedt ${ }^{1}$, and L. Eliasson ${ }^{2}$ \\ ${ }^{1}$ Swedish Institute of Space Physics, Lund, Sweden \\ ${ }^{2}$ Swedish Institute of Space Physics, Kiruna, Sweden \\ Received: 22 February 2005 - Revised: 6 July 2005 - Accepted: 13 July 2005 - Published: 22 November 2005 \\ Part of Special Issue "1st European Space Weather Week (ESWW)"
}

\begin{abstract}
The 7-10 November 2004 period contains two events for which the local ground magnetic field was severely disturbed and simultaneously, the solar wind displayed several shocks and negative $B_{z}$ periods. Using empirical models the 10-min RMS $\Delta X$ and $\Delta Y$ at Brorfelde (BFE, $11.67^{\circ} \mathrm{E}$, $55.63^{\circ} \mathrm{N}$ ), Denmark, are predicted. The models are recurrent neural networks with 10-min solar wind plasma and magnetic field data as inputs. The predictions show a good agreement during 7 November, up until around noon on 8 November, after which the predictions become significantly poorer. The correlations between observed and predicted log RMS $\Delta X$ is 0.77 during 7-8 November but drops to 0.38 during 9-10 November. For RMS $\Delta Y$ the correlations for the two periods are 0.71 and 0.41 , respectively. Studying the solar wind data for other L1-spacecraft (WIND and SOHO) it seems that the ACE data have a better agreement to the near-Earth solar wind during the first two days as compared to the last two days. Thus, the accuracy of the predictions depends on the location of the spacecraft and the solar wind flow direction. Another finding, for the events studied here, is that the $\Delta X$ and $\Delta Y$ models showed a very different dependence on $B_{z}$. The $\Delta X$ model is almost independent of the solar wind magnetic field $B_{z}$, except at times when $B_{z}$ is exceptionally large or when the overall activity is low. On the contrary, the $\Delta Y$ model shows a strong dependence on $B_{z}$ at all times.
\end{abstract}

Keywords. Magnetospheric physics (Solar windmagnetosphere) - Geomagnetism and Paleomagnetism (Rapid time variations) - Ionosphere (Modeling and forecasting)

\section{Introduction}

The Earth's magnetosphere is a dynamic system that responds to changes in the upstream solar wind. Through complex processes that includes magnetic reconnection and viscous instabilities, energy is transferred from the solar wind into the magnetosphere (Baumjohann and Haerendel, 1987), with subsequent energy dissipation through geomagnetic storms and substorms (Gonzalez et al., 1994). A major fraction of large geomagnetic storms is caused by coronal mass ejections (CME) (Gosling et al., 1991). The CME, and its interplanetary counterpart, the ICME, plows through the ambient solar wind, producing shock waves and following sheath regions (Owens et al., 2005). In some cases the ICME evolves as a magnetic cloud (Burlaga, 1995) with smooth magnetic field line rotation during which the $B_{z}$ component may be strongly negative for an extended period of time, enabling entry of solar wind energy through magnetic reconnection. Another source for geomagnetic activity, especially during the declining phase of solar activity, is seen in high speed solar wind streams (Richardson et al., 2002). The different structures interact and evolve as they travel from the Sun to the Earth, causing various degrees of geoeffectiveness (Huttunen et al., 2002; Echer and Gonzales, 2004).

During the geomagnetic storm, different current systems are modified, like the ionospheric currents, ring current, and magnetopause current. On the ground the currents are observed as deviations of the local geomagnetic field (Nishida, 1978). Several indices have been derived for various geophysical phenomena (Mayaud, 1980) and their coupling to the solar wind have been extensively studied (Baker, 1986), and especially the $D_{s t}$ index (Wu and Lundstedt, 1997; Klimas et al., 1998). The effects of geomagnetic disturbances are observed on technological systems, such as electrical power grids, pipe lines, and telegraph lines (Boteler et al., 1998, Lundstedt, 2004 ${ }^{1}$ ), and are called geomagnetically

\footnotetext{
${ }^{1}$ Lundstedt, H.: The Sun, Space Weather and GIC Effects in Sweden, Adv. Space Res, in review, 2004.
}

Correspondence to: $\mathrm{P}$. Wintoft

(peter@lund.irf.se) 
induced currents (GIC). There is great interest in modelling GIC, both for post-event analysis and for predictions. As a result there are three parallel GIC studies within the ESA Space Weather Applications Pilot Project and these can be found at the web page http://www.esa-spaceweather.net/.

The GIC can be estimated in different ways. One approach is to use geomagnetic indices, as several can be successfully predicted: $A E$ (Gleisner and Lundstedt, 2001a), $D_{s t}$ (Vassiliadis and Klimas, 1999; Lundstedt et al., 2002), and $K_{p}$ (Boberg et al., 2000). The index is then translated into a physical quantity that is related to GIC. Boteler (2001) showed that there is close to a linear relationship between the 3-h $K_{p}$ index and the logarithm of the ground electric field. However, the indices have their limitations because they have been derived to capture some specific aspect of the magnetospheric variation. Another approach is to use observed ground geomagnetic field data. The calculation of GIC can then be divided into two steps (Pirjola, 2002) involving a geophysical part to determine the geoelectric field and an engineering part to compute the currents in the technological system. The electric field is computed from the magnetic field by assuming an equivalent ionospheric current system such that the geomagnetic variations at the Earth's surface can be explained by horizontal divergence-free ionospheric currents (Viljanen et al., 2003). Solar wind-magnetosphere coupling models can then be used to predict the local ground magnetic field. In Gleisner and Lundstedt (2001b) a model was developed that predicts the 10-min average local geomagnetic field using solar wind data. But, as the electric field is related to the rate-of-change of the magnetic field $(d B / d t)$ via Faraday's law of induction $\nabla \times E=-\frac{\partial B}{\partial t}$, a more basic quantity to use is the time difference of $B$, i.e. $\Delta B(t)=B(t+1)-B(t)$ (Viljanen et al., 2001). However, most of the power in $\Delta B$ is located at small scales (high frequencies) and therefore a large fraction of the signal will be lost if $\Delta B$ is temporally averaged, or if $\Delta B$ is formed from a temporally averaged $B$ (Wintoft, 2005). This happens already at 5 to $10 \mathrm{~min}$ averages. Therefore, other moments of $\Delta B$ should be considered. In the work by Weigel et al. (2002) models were developed that predict the average absolute value of $\Delta B$ with a temporal resolution of $30 \mathrm{~min}$. More specifically, they studied the north-south component of the magnetic field, i.e. $\langle|\Delta X|\rangle_{30 \mathrm{~min}}$. As the average is taken of the absolute value, a large fraction of the variance from the original signal is maintained. The best model reached an overall prediction efficiency of 0.4 based on data from 1998-1999.

The models developed by Wintoft (2005) aims instead at predicting the 10-min root-mean-square (RMS) of $\Delta X$ (and $\Delta Y$ ). The motivation of using RMS data is summarised here. The power spectrum of $\Delta X$ peaks at small scales and decreases quickly with increasing scale: $83 \%$ of the power is located at scales $\tau \leq 8 \mathrm{~min}, 99 \%$ at $\tau \leq 128 \mathrm{~min}$. We speak in terms of scales as defined from wavelet analysis, but the scale may be translated into the approximate frequency band $[1 / 4 \tau, 1 / 2 \tau]$ (Percival and Walden, 2002). One may also picture the signal at a certain scale as being the difference between two consecutive averages of width $\tau$. It was found that the RMS data can be used to estimate the power spectra of $\Delta X$ and $\Delta Y$. This is useful for the subsequent analysis, for example, computing GIC, as both amplitude and scale (frequency) are available. Another issue is that the RMS data captures a major fraction of the variance in $\Delta X$. The relative variance is $\operatorname{Var}(\operatorname{RMS} \Delta X) / \operatorname{Var}(\Delta X)=0.82$. For comparison the 10-min average absolute $\Delta X$ has a relative variance of $\operatorname{Var}(\langle|\Delta X|\rangle) / \operatorname{Var}(\Delta X)=0.55$. Finally, any temporal averaging will decrease the forecast lead time. To illustrate this we may consider a time dependent parameter $x(t)$ that is collected with a sampling interval $\Delta t$ that results in the time series $x_{i}$. The corresponding time stamp $t_{i}$ marks the beginning of the interval so that $x_{i}$ is the average of $x(t)$ over the interval $t \in\left[t_{i}, t_{i+1}\right]$, where $t_{i+1}=t_{i}+\Delta t$. Similarly, we may have another variable $y(t)$ sampled to $y_{i}$. If we now wish to develop a model that predicts $y$ from $x$ with lead time $T$ we have $\hat{y}(t+T)=f(x(t))$, where $\hat{y}$ is the prediction of $y$. This leads to the discrete model $\hat{y}_{i+k}=f\left(x_{i}\right)$ where $T=k \Delta t$. Now assume that the current time is $t_{0}$. The latest available input is $x_{-1}$ and it has been collected over the time interval $\left[t_{-1}, t_{0}\right]$. With a forecast time of $T=k \Delta t$ we will therefore forecast $y_{k-1}$, resulting in a true forecast time of $T^{\prime}=T-\Delta t$. In order for the model to perform actual forecasts, we must have $\Delta t \leq T$.

In this work we will use the previously developed models to predict the disturbed period during 7 to 10 November 2004. The following section describes the observed data, Sect. 3 describes the model, Sect. 4 address forecast errors related to the location of the solar wind monitor and the control of the IMF $B_{z}$ component. In Sect. 5 the conclusions are given.

\section{The 7-10 November 2004 events}

The 7-10 November 2004 period contains two events for which the local ground magnetic field was severely disturbed and the solar wind displays several shocks and negative $B_{z}$ periods. In Fig. 1 the solar wind plasma and magnetic field data are shown, together with the ground magnetic field deviations at Brorfelde (BFE, $\left.11.67^{\circ} \mathrm{E}, 55.63^{\circ} \mathrm{N}\right)$. The deviations are the one-minute differences of the north-south $(X)$ and east-west $(Y)$ magnetic field components

$$
\begin{aligned}
& \Delta X(t)=X(t+1)-X(t), \\
& \Delta Y(t)=Y(t+1)-Y(t),
\end{aligned}
$$

as approximations to $d X / d t$ and $d Y / d t$. The solar wind data comes from three spacecraft: ACE (blue line), WIND (green line), and SOHO (red dots). The ACE (Smith et al., 1998; McComas et al., 1998) and WIND (Ogilvie et al., 1995) data have been resampled to 10-min averages while the SOHO (Ipavich et al., 1998) data are one-hour averages. The four top panels show the particle density $n$, the standard deviation $\sigma_{n}$ of the density, the $B_{z}$ magnetic field component in GSM, and the velocity $V$. The next two panels show the one-minute differences $\Delta X$ and $\Delta Y$. The two panels also contain the 
10-min root-mean-square (RMS) of $\Delta X$ and $\Delta Y$. The bottom panel shows the north-south magnetic field $X$, minus $17150 \mathrm{nT}$, and the $D_{s t}$ index. The five vertical lines indicates the times of solar wind shocks. It is clear that these shocks are followed by sudden increases in $|\Delta X|$ and $|\Delta Y|$. As the geomagnetic storm develops, large variations in $\Delta X$ and $\Delta Y$ are seen. During 8 November, the extreme values reach $(\Delta X, \Delta Y)=(140,116) \mathrm{nT} / \mathrm{min}$ and during 9 November $(\Delta X, \Delta Y)=(-242,-229) \mathrm{nT} / \mathrm{min}$. The corresponding 10 -min RMS extreme values are $(96,61) \mathrm{nT} / \mathrm{min}$ and $(122$, 104) $\mathrm{nT} / \mathrm{min}$, respectively. We also note that the ratio RMS $\Delta X /$ RMS $\Delta Y$ decreases from 1.6 for 8 November event to 1.2 for the 9 November event, indicating that the disturbance is more along the north-south direction during the first event. These events have not yet been described in the scientific literature, however, a description can be found at Space Environment Center (http://www.sec.noaa.gov/weekly/).

We see that the Sun was very active with several CMEs. The first shock in early 7 November was probably caused by a CME on 3 November. There was a small increase in the solar wind magnetic field with a negative $B_{z}$ component. Both $\Delta X$ and $\Delta Y$ display an impulse $66 \mathrm{~min}$ later in accordance with the ACE-magnetopause travel time of $t_{\mathrm{ACE}}=68 \mathrm{~min}$ at the velocity of $365 \mathrm{~km} / \mathrm{s}$. The second shock, caused by a CME on 4 November was accompanied with larger increases in particle density $n, \sigma_{n}$, and $B . B_{z}$ turned initially northward and later southward. The shock was followed by a magnetic impulse 55 min later, again in agreement with the $420 \mathrm{~km} / \mathrm{s}$ velocity $\left(t_{\mathrm{ACE}}=59 \mathrm{~min}\right)$. Both $\Delta X$ and $\Delta Y$ continued to be slightly disturbed and $D_{s t}$ showed a weak increase followed by a weak decrease typical for the magnetic storm initial and main phases. At the third shock the velocity jumped from $500 \mathrm{~km} / \mathrm{s}$ to above $650 \mathrm{~km} / \mathrm{s}$, the magnetic field increased to almost $50 \mathrm{nT}$ and $B_{z}$ turned initially northward and later strongly southward for an extended period of time. The source for this event was probably a series of CMEs that occurred late on 4 and early 6 November. The magnetic impulse took place 33 minutes later, in good agreement with a velocity of $650 \mathrm{~km} / \mathrm{s}\left(t_{\mathrm{ACE}}=38 \mathrm{~min}\right)$. The disturbed period continued for about $19 \mathrm{~h}$ and $D_{s t}$ reached $-373 \mathrm{nT}$ on 8 November. What looks like a magnetic impulse early in 9 November is most likely a spurious value, as there are data gaps around that point and other stations show no such feature. The fourth shock, around noon on 9 November shows quite different velocities for ACE/WIND and SOHO. The magnetic impulse occurs 16 min after the shock and is similar in strength to that after the second shock. With a velocity of $V_{\mathrm{ACE}}=790 \mathrm{~km} / \mathrm{s}$ there should be a delay of $t_{\mathrm{ACE}}=32 \mathrm{~min}$. It is thus difficult to associate this magnetic impulse with the measured solar wind at ACE. The fourth shock shows a jump in velocity from $650 \mathrm{~km} / \mathrm{s}$ to $800 \mathrm{~km} / \mathrm{s}$ and a magnetic impulse follows $29 \mathrm{~min}$ later, in agreement with $t_{\mathrm{ACE}}=31 \mathrm{~min}$.

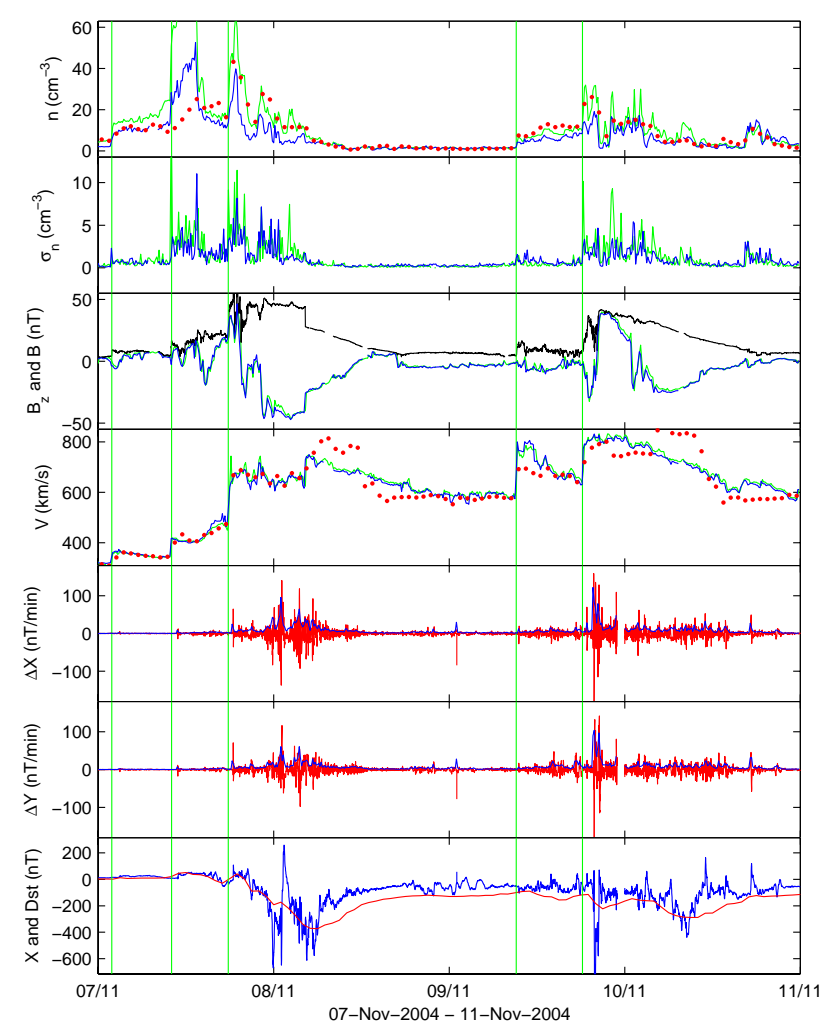

Fig. 1. The panels show, from top to bottom: solar wind density $n$ (ACE-blue, WIND-green, SOHO-red), standard deviation $\sigma_{n}$ of the density, $B_{z}$ magnetic field component in GSM and total field $B$ from ACE (black), velocity $V$, one-minute $\Delta X$ (red) and 10-min RMS $\Delta X$ (blue), one-minute $\Delta Y$ and 10-min RMS $\Delta Y$, and one minute $X$ (blue) and hourly $D_{s t}$ (red). The period extends over the four days of 7-10 November 2004. The only available data from SOHO are the hourly average density and velocity.

\section{Forecasting RMS $(\Delta X, \Delta Y)$ using ACE}

The empirical models previously developed predict the 10min RMS $\Delta X$ and $\Delta Y$ for southern Scandinavia, with a prediction lead time of $30 \mathrm{~min}$ (Wintoft, 2005). The models are recurrent neural networks with solar wind plasma and magnetic field data as inputs: 10-min averages of magnetic field $B_{z}$, particle density $n$, velocity $V$, and standard deviations $(\sigma)$ of the same parameters. Local time and time of year were also used.

The models were trained and validated on data from the six year period 1998-2003. As the distributions of RMS $\Delta X$ and $\Delta Y$ are dominated by values close to zero only a selected subset was used, in order to avoid the network becoming biased towards quiet conditions. However, large values are still typically underestimated, as they are more infrequent. The prediction horizon of 30 min was selected to enable the models to predict events with a large range of solar wind velocities. A velocity of $830 \mathrm{~km} / \mathrm{s}$ at L1 takes $30 \mathrm{~min}$ to reach the magnetopause. We also studied models where the prediction lead time was increased up to $90 \mathrm{~min}$, but for both $\Delta X$ and $\Delta Y$ the correlation decreased. A large set of neural 


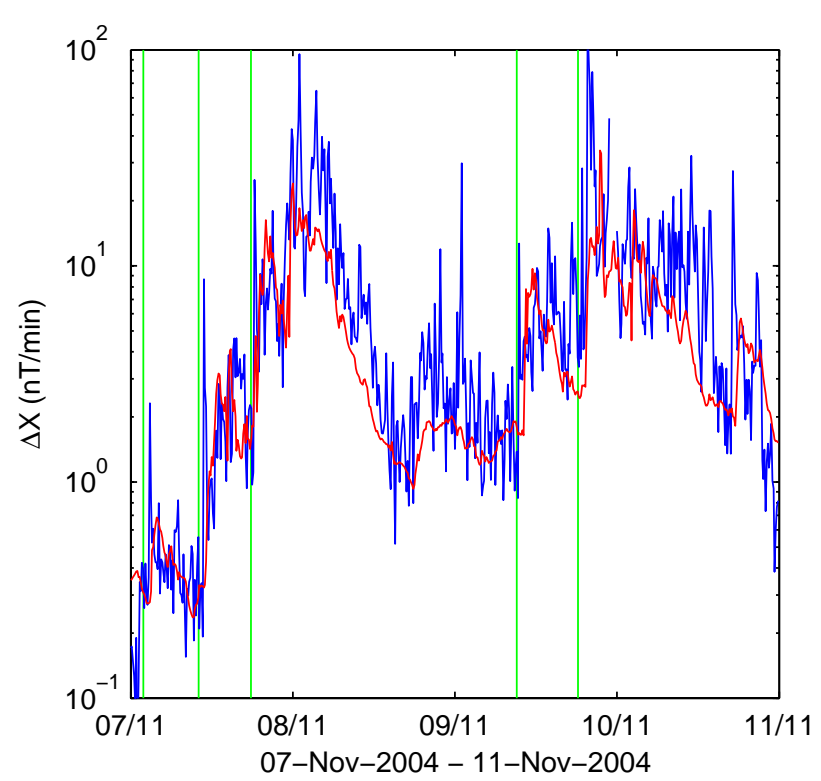

Fig. 2. The figure shows the observed (blue) and predicted (red) 10-min RMS $\Delta X$.

networks were trained and the optimal models gave a correlation of 0.79 and prediction efficiency (Detman and Vassiliadis, 1997) of 0.63 of the logarithm of the RMS data. It is important to notice here that the geomagnetic data are not used as input to the model, only solar wind data, otherwise the correlation could be even higher but not necessarily truly improving the predictions. For example, a simple persistence model, predicting RMS $\Delta X(t+30 \mathrm{~min})$ based on RMS $\Delta X(t)$, would have a correlation of 0.72 but the predictions would consistently lag by $30 \mathrm{~min}$. To verify that the solar wind $-\Delta X$ model is actually making $30 \mathrm{~min}$ forecasts, with the stated correlation, we may compute the correlation coefficient between the observed $\Delta X$ and the predicted $\Delta X$ by shifting the predicted $\Delta X$ backwards and forwards in time. For a true forecast the maximum correlation should occur at $30 \mathrm{~min}$ and decrease for smaller and larger prediction times, and this is also the case for the neural network models.

It was shown that the solar wind influence on $\Delta X$ and $\Delta Y$ were slightly different. The most important inputs for $\Delta X$, in order of increasing importance, were local time, $B_{z}$, $\sigma_{n}$, and $V$. For $\Delta Y$ it was local time, $\sigma_{n}, V$, and $B_{z}$. The other inputs, and mots notably $n$, had no significant influence. The independence of $n$ was also shown by Weigel et al. (2002). The models have been implemented for real time operation and the forecasts are displayed on a web page (http://www.lund.irf.se/gicpilot/gicforecastprototype/). The predictions of RMS $\Delta X$ and $\Delta Y$ for the November events are shown in Figs. 2 and 3. It is seen that the predictions capture the large-scale variations but not the sample-to-sample variations. The predictions show a good agreement during 7 November, up until around noon on 8 November, after which the predictions become significantly poorer. The correlations between observed and predicted $\log$ RMS $\Delta X$ is 0.77 during

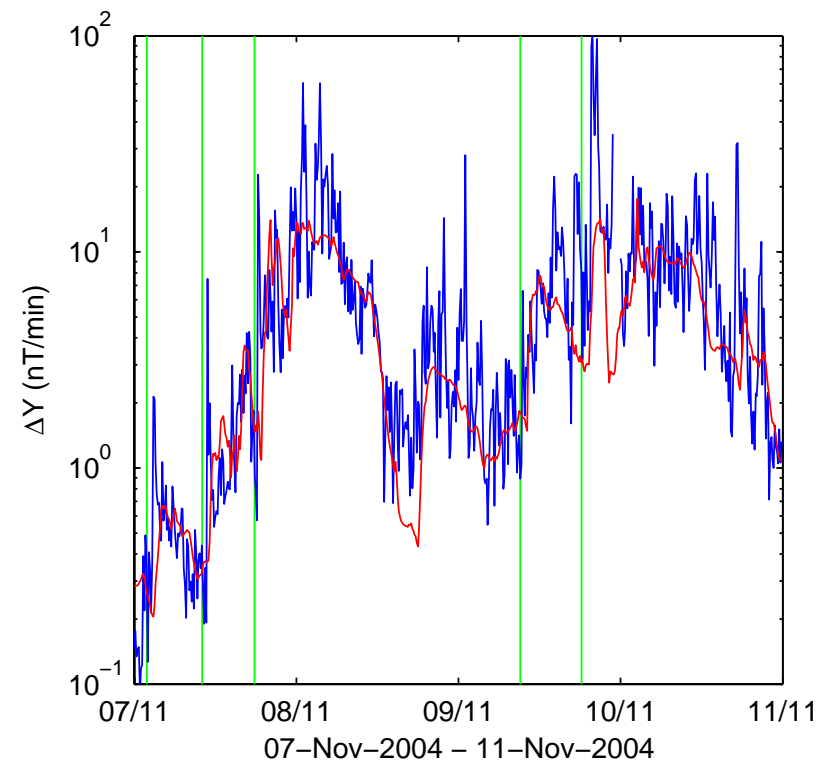

Fig. 3. The figure shows the observed (blue) and predicted (red) 10-min RMS $\Delta Y$.

7-8 November but drops to 0.38 during $9-10$ November. For RMS $\Delta Y$ the correlation for the two periods are 0.71 and 0.41 , respectively.

\section{Discussion}

In the following sections we address the forecast quality in relation to the location of the solar wind monitor and study the coupling to the solar wind $B_{z}$ component.

\subsection{The locations of solar wind monitors}

The ACE spacecraft is in orbit around L1 approximately 240 Earth radii $\left(\mathrm{R}_{\mathrm{E}}\right)$ upstream from Earth. Due to the large distance there may be considerable differences in solar wind properties at ACE and close to Earth. In the study by Dalin et al. (2002) it was shown that the correlation of solar wind plasma data from different spacecraft could, at times, be small and the correlation decreased with increasing (Y,Z)separation. For the period studied here the ACE spacecraft is located approximately at $(\mathrm{X}, \mathrm{Y}, \mathrm{Z})=(242,23,-15) R_{E}$ in GSE coordinate system. Two other spacecraft are also located around L1, namely SOHO at $(218,-104,-3) R_{E}$ and WIND at $(199,59,-9) R_{E}$. The spacecraft are almost located in the $(\mathrm{X}, \mathrm{Y})$-plane, with the largest separation of $12 R_{E}$ in the Zdirection. However, in the Y-direction the distance is as large as $163 R_{E}$. In the X-direction the separation is about $42 R_{E}$, and with a velocity of $400 \mathrm{~km} / \mathrm{s}$ or higher the separation in time is less than 11 minutes. From Fig. 1 we see that the $B_{z}$ magnetic field components for ACE and WIND are very well correlated. There is a shift of $<10 \mathrm{~min}$, barely visible in the figure, that comes from the separation in X. Studying the velocity, there is again a very good agreement between ACE 


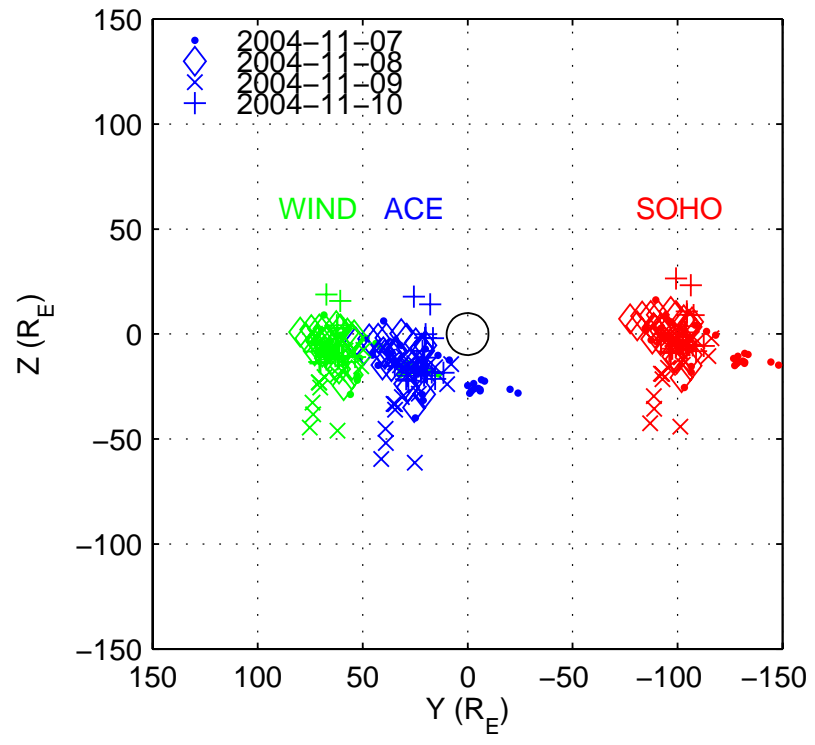

Fig. 4. The figure shows the advected spacecraft locations onto the plane $\mathrm{X}=10 R_{E}$ using hourly average velocity vectors from WIND. The data have been divided into the four days: 7 November (dots), 8 November (diamonds), 9 November (crosses), and 10 November (pluses). The black circle is centred on Earth and has a radius of $10 R_{E}$.

and WIND. The hourly average SOHO velocity data show a good agreement during 7 November, up to 05:00 UT on 8 November, despite its large distance from ACE and WIND. After that, the SOHO velocity deviates significantly most of the time. It thus seems that the velocity structure is homogenous at the beginning of the period and then becomes more fragmented. The ACE and WIND densities show similar temporal variation, although WIND mostly gives higher densities, often by a factor 2 to 3 . Except for the first part of 7 November, the SOHO density agrees quite well with the WIND density.

In Fig. 4 the crossing of the solar wind in the (Y, Z)-plane at $\mathrm{X}=10 R_{E}$ (typical megnetopause distance) is shown for the three spacecraft. As we only have all three velocity components for WIND, we have assumed the same velocity for the three spacecraft. The circle in the centre has a radius of $10 R_{E}$. We see that the measurements at ACE advected to $X=10$ come reasonably close to the Earth during 7 November (dots). The most distant points are the two westerly dots, but simultaneously the WIND measurements come close to the Earth, and as ACE and WIND show a very good agreement for the whole period, we may conclude that the measurements at ACE depicts quite well what the solar wind is like close to the Earth during 7 November. Then, during 8 November, the advected location of ACE (diamonds) moves towards the east and south. During this day the velocities at SOHO and ACE also start to deviate. Then, during 9 November, the advected ACE location turns far south (crosses) of the Earth. It is thus possible that the measurements at ACE from the morning of 8 November through 9 November do

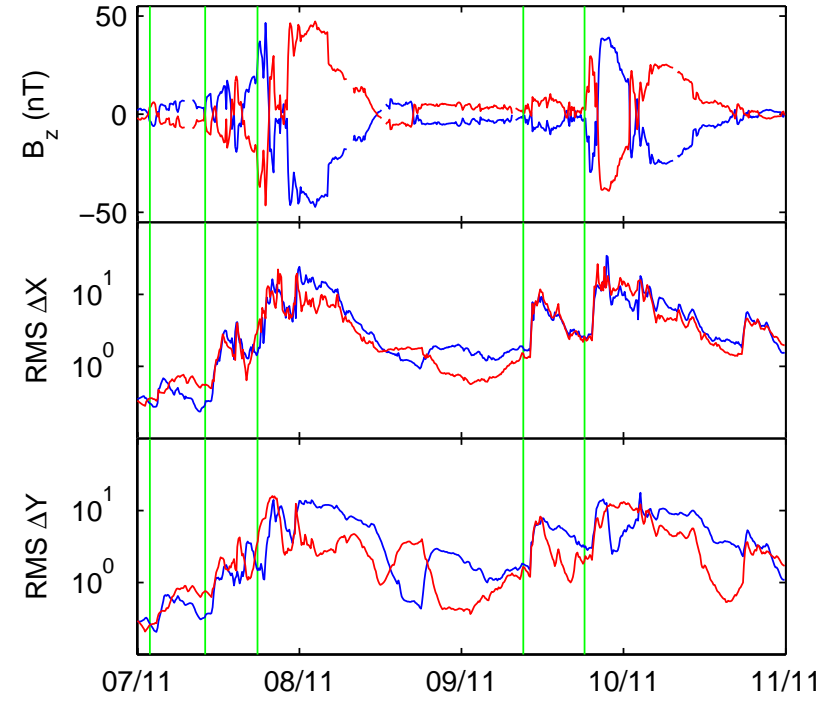

Fig. 5. The three panels show, from top to bottom: solar wind $B_{z}$ (blue) and $-B_{z}$ (red), predicted RMS $\Delta X$ using $B_{z}$ (blue) and $-B_{z}$ (red), and predicted RMS $\Delta Y$ using $B_{z}$ (blue) and $-B_{z}$ (red).

not represent accurately the solar wind close to the Earth.

Turning back to the predictions shown in Figs. 2 and 3, it was seen that the correlation was higher during the first two days as compared to the last two days. Thus, this may be the result that the solar wind at ACE correlates well with the solar wind close to the Earth in the early part of the period but not later in the period. The errors are particularly large during the late evening to midnight on 9 November. Studying another model that predicts the geomagnetic index $D_{s t}$ (Lundstedt et al., 2002), also using ACE data as input, reveals that the prediction errors are large for the same hours.

\subsection{The influence of $B_{z}$}

Using the prediction models the influence of the solar wind on RMS $\Delta X$ and $\Delta Y$ may be studied. In principal, some interesting artificial values could be selected to represent the solar wind to study the response of the model. However, care has to be taken so that the data represent a valid physical and statistical configuration, otherwise the output from the model will not be correct. One parameter that can be easily studied is $B_{z}$. It is perfectly valid to change the sign on $B_{z}$ and run the models, as we expect that the direction of $B$ is not correlated with density or velocity. For example, a magnetic cloud starts with a simultaneous increase in $n, V$, and $B$ (Burlaga, 1995). But $B_{z}$ may initially either turn northward or southward, with the subsequent evolution determined by the cloud topology. The result is shown in Fig. 5. The blue curve represents the original $B_{z}$ and the red curve $-B_{z}$ (top panel). The model outputs are shown accordingly, in blue and red (bottom panels). The first apparent observation is that RMS $\Delta X$ shows a weak coupling to $B_{z}$, as changing the sign has very little effect on the output. On the contrary, RMS $\Delta Y$ is much more affected. 
Looking at the details we note that the variations in the two predictions of RMS $\Delta X$ are very similar up to 23:00 UT on 7 November, even though the sign on $B_{z}$ has been changed. During the following hours, when $B_{z}$ is strongly negative, up to $05: 00$ UT on 8 November there is a difference of about a factor 2 . Then, the predictions coincide again when $B_{z}$ goes towards zeros and the velocity decreases. But then, during a period of low activity from 12:00 UT on 8 November to 09:00 UT on 9 November, when $B_{z}$ is close to zero, there is again a difference of a factor 2 , with slightly higher activity when $B_{z}$ is negative.

\section{Conclusions}

In this work we have studied the prediction of the 10-min variation of the local ground magnetic field, more specifically, the 10-min RMS $\Delta X$ and $\Delta Y$. The prediction model uses the solar wind data from the ACE spacecraft. The fourday period extending over 7 November to 10 November 2004 was explored.

It was found that the sample-to-sample variations in RMS $\Delta X$ and $\Delta Y$ are not predicted, but the large-scale variations are predicted. The predictions of the first two days show a higher correlation with the observations than during the last two days. By studying the solar wind data for other L1spacecraft (WIND and SOHO), it seems that the ACE data have a better agreement to the near-Earth solar wind during the first two days as compared to the last two days. In a study by Dalin et al. (2002) it was also shown that the correlation of solar wind plasma data from different spacecraft decreased with increasing (Y, Z)-separation. Thus, the accuracy of the predictions depends on the location of the spacecraft and the solar wind flow direction.

The models have not been developed to predict the response of $\Delta X$ or $\Delta Y$ for specific solar wind structures; the only criterion used on the data selection is that there should be contiguous data for $48 \mathrm{~h}$, or longer, for which RMS $>10 \mathrm{nT} / \mathrm{min}$ at least once. This means that solitary peaks in RMS $\Delta X$ or $\Delta Y$ will be difficult to model because of the low relative occurrence rate. However, the peaks may also be related to magnetic impulse events (Kataoka et al., 2003) that show a significant correlation to discontinuities of the interplanetary magnetic field, and not $B_{z}$, therefore, additional inputs should be considered in future studies.

By modifying the solar wind input data the response of the model may be studied. Care has to be taken in how the input is modified, but a valid modification from a physical and statistical point of view is to change the sign of $B_{z}$. It was found, for the events studied here, that the $\Delta X$ and $\Delta Y$ models showed a very different dependence on $B_{z}$. The $\Delta X$ model is almost independent of the solar wind magnetic field $B_{z}$, except at times when $B_{z}$ is large or when the overall activity is low. On the contrary, the $\Delta Y$ model shows a strong dependence on $B_{z}$ at all times.

In the models developed by Wintoft (2005) the location of ACE was not considered. Thus, it is reasonable to believe that there are data in the solar wind data set used for model development that are poorly correlated to the near-Earth solar wind. The inclusion of such data during the model development has the effect of increased noise. In future work, a more careful selection of data, taking into account the location of ACE, should be considered.

Acknowledgements. We are grateful to the providers of the solar wind and ground magnetic field data: ACE data from CalTech and SEC; the MIT Space Plasma Group for the WIND plasma data and NASA/GSFC for the WIND magnetic field data; the University of Maryland for the SOHO/PM data; the Solar-Terrestrial Physics Division at the Danish Meteorological Institute for the Brorfelde magnetic field data. COST 724 is acknowledged for financial support. This work has been carried out with support from ESA/ESTEC (Contract No. 16953/02/NL/LvH).

Topical Editor T. Pulkkinen thanks J. B. Blake and another referee for their help in evaluating this paper.

\section{References}

Baker, D. N.: Statistical analyses in the study of solar windmagnetosphere coupling, Solar Wind-Magnetosphere Coupling, 17-38, 1986.

Baumjohann, W. and Haerendel, G.: Entry and dissipation of energy in the Earth's magnetosphere, in Space Astronomy and Solar System Exploration: Proceeding of summer school held at aplbach, Austria, 29 July-8 August, 1986, 121-130, ESA, 1987.

Boberg, F., Wintoft, P., and Lundstedt, H.: Real time $K_{p}$ predictions from solar wind data using neural networks, Physics and Chemistry of the Earth, 25, 275-280, 2000.

Boteler, D.: Assessment of geomagnetic hazards to power systems in Canada, Natural Hazards, 23, 101-120, 2001.

Boteler, D. H., Pirjola, R. J., and Nevanlinna, H.: The effects of geomagnetic disturbances on electrical systems at the Earth's surface, Adv. Space Res., 22, 17-27, 1998.

Burlaga, L. F.: Interplanetary magnetohydrodynamics, Oxford University Press, 1995.

Dalin, P., Zastenker, G., Paularena, K., and Richardson, J.: The main features of solar wind plasma correlations of importance to space weather strategy, J. Atmos. S.-P., 64, 737-742, 2002.

Detman, T. R. and Vassiliadis, D.: Review of techniques for magnetic storm forecasting, 253-266, AGU, 1997.

Echer, E. and Gonzales, W. D.: Geoeffectiveness of interplanetary shocks, magnetic clouds, sector boundary crossings and their combined occurrence, Geophys. Res. Lett., 31, L09 808, 2004.

Gleisner, H. and Lundstedt, H.: Auroral electrojet predictions with dynamic neural networks, J. Geophys. Res., 106, 24 541-24 550, 2001a.

Gleisner, H. and Lundstedt, H.: Neural network-based local model for prediction of geomagnetic disturbances, J. Geophys. Res., 106, 8425-8433, $2001 b$.

Gonzalez, W. D., Joselyn, J. A., Kamide, Y., Kroehl, H. W., Rostoker, G., Tsurutani, B. T., and Vasyliunas, V. M.: What is a geomagnetic storm?, J. Geophys. Res., 99, 5771-5792, 1994.

Gosling, J. T., McComas, D. J., Phillips, J. L., and Bame, S. J.: Geomagnetic activity associated with Earth passage of interplanetary shock disturbances and coronal mass ejections, J. Geophys. Res., 96, 7831-7839, 1991. 
Huttunen, K. E. J., Koskinen, H. E. J., and Schwenn, R.: Variability of magnetospheric storms driven by different solar wind pertubations, J. Geophys. Res., 107, SMP 20-1-8, 2002.

Ipavich, F. M., Galvin, A. B., Lasley, S. E., Paquette, J. A., Hefti, S., Reiche, K., Coplan, M. A., Gloeckler, G., Bochsler, P., Hovestadt, D., Grünwaldt, H., Hilchenbach, M., et al.: Solar wind measurements with SOHO: The CELIAS/MTOF proton monitor, J. Geophys. Res., 103, 17 205-17 213, 1998.

Kataoka, R., Fukunishi, H., and Lanzerotti, L. J.: Statistical identification of solar wind origins of magnetic impulsive events, J. Geophys. Res., 108, SMP 13-1-12, 2003.

Klimas, A. J., Vassiliadis, D., and Baker, D. N.: $D_{s t}$ index prediction using data-derived analogues of the magnetospheric dynamics, J. Geophys. Res., 103, 20 435-20 448, 1998.

Lundstedt, H., Gleisner, H., and Wintoft, P.: Operational forecasts of the geomagnetic Dst index, Geophys. Res. Lett., 29, 34-134-4, 2002.

Mayaud, P. N.: Derivation, meaning, and use of geomagnetic indices, Geophysical Monograph Series, AGU, 1980.

McComas, D. J., Bame, S. J., Barker, P., Feldman, W. C., Phillips, J. L., Riley, P., and Griffee, J. W.: Solar wind electron proton alpha monitor (SWEPAM) for the Advanced Composition Explorer, Space Science Reviews, 86, 563-612, 1998.

Nishida, A.: Geomagnetic Diagnosis of the Magnetosphere, 9 of Physics and Chemistry in Space, Springer-Verlag, 1978.

Ogilvie, K. W., Chornay, D. J., Fritzenreiter, R. J., Hunsaker, F., Keller, J., Lobell, J., Miller, G., Scudder, J. D., Sittler, Jr., E. C., Torbert, R. B., Bodet, D., Needell, G., Lazarus, A. J., et al.: SWE, a comprehensive plasma instrument for the WIND spacecraft, Sp. Sc. R., 71, 55-77, 1995.

Owens, M. J., Cargill, P. J., Pagel, C., Siscoe, G. L., and Crooker, N. U.: Characteristic magnetic field and speed properties of interplanetary coronal mass ejections and their sheath regions, J. Geophys. Res., 110(A01), 105, 2005.

Percival, D. B. and Walden, A. T.: Wavelet methods for time series analysis, Cambridge University Press, 2002.
Pirjola, R.: Review on the calculation of surface electric and magnetic fields and of geomagnetically induced currents in groundbased technological systems, Surv. Geophys., 23, 71-90, 2002.

Richardson, I. G., Cane, H. V., and Cliver, E. W.: Sources of geomagnetic activity during nearly three solar cycles, J. Geophys. Res., 107, SSH 8-1-13, 2002.

Smith, C. W., L'Heureux, J., Ness, N. F., Acuña, M. H., Burlaga, L. F., and Scheifele, J.: The ACE magnetic fields experiment, Space Sci. R., 86, 613-632, 1998.

Vassiliadis, D. and Klimas, A. J.: The $D_{s} t$ geomagnetic response as a function of storm phase and amplitude and the solar wind electric field, J. Geophys. Res., 104, 24 957-24 976, 1999.

Viljanen, A., Nevanlinna, H., Pajunpää, K., and Pulkkinen, A.: Time derivate of the horizontal geomagnetic field as an activity indicator, Ann. Geophys., 19, 1107-1118, 2001,

SRef-ID: 1432-0576/ag/2001-19-1107.

Viljanen, A., Pulkkinen, A., Amm, O., Pirjola, R., Korja, T., and BEAR Working Group: Fast computation of the geoelctric field using the method of elementary current systems and planar Earth models, Ann. Geophys., 22, 101-113, 2004,

SRef-ID: 1432-0576/ag/2004-22-101

Weigel, R. S., Vassiliadis, D., and Klimas, A. J.: Coupling of the solar wind to temporal fluctuations in ground magnetic fields, Geophys. Res. Lett., 29, 2002.

Wintoft, P.: Study of the Solar Wind Coupling to the Time Difference Horizontal Geomagnetic Field, Ann. Geophys., 23, 19491957, 2005,

SRef-ID: 1432-0576/ag/2005-23-1949.

Wu, J.-G. and Lundstedt, H.: Neural network modeling of solar wind-magnetosphere interaction, J. Geophys. Res., 102, 14457 14466, 1997. 